\title{
Sugiura modificado: una alternativa para pacientes no cirróticos con trombosis de la vena porta extrahepática y sangrado variceal recurrente
}

\section{Modified sugiura procedure: an alternative for non-cirrhotic patients with extrahepatic portal vein thrombosis and recurrent variceal bleeding}

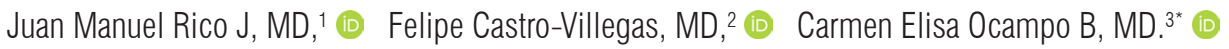

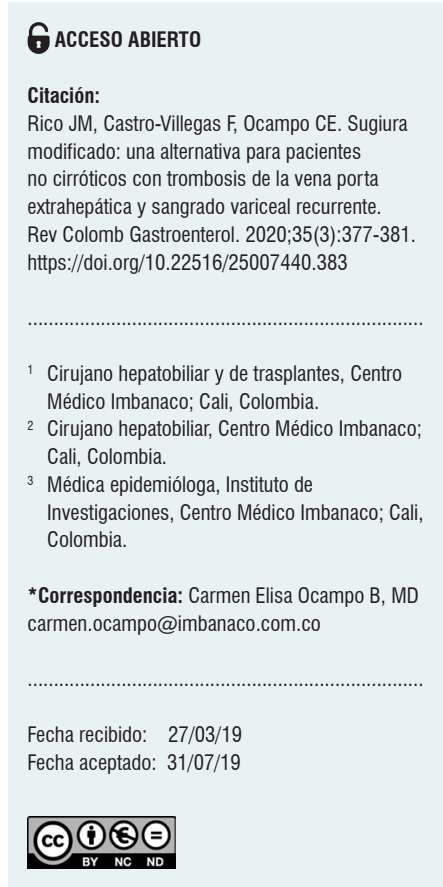

\begin{abstract}
Resumen
Una de las consecuencias más graves de la trombosis de la vena porta extrahepática es la hipertensión portal con sangrado variceal recurrente. Una vez falla la ligadura endoscópica de las várices y el eje esplenoportal no se encuentra permeable, la devascularización tipo Sugiura modificado puede ser la única alternativa. Se ha documentado su uso en pacientes con cirrosis, pero hay poca información en personas no cirróticas. En este artículo se describe una serie de 4 casos de pacientes no cirróticos, en los cuales se realizó dicho procedimiento. Los pacientes fueron seguidos durante 12 meses y ninguno presentó episodios de resangrado de las várices esofágicas, ni tampoco se requirió la ligadura de las várices residuales. Esta cirugía se perfila como una alternativa terapéutica para este tipo de pacientes.
\end{abstract}

\section{Palabras clave}

Trombosis, vena porta extrahepática, hipertensión portal, Sugiura modificado, várices esofágicas, cirrosis

\section{Abstract}

One of the most serious consequences of extrahepatic portal vein thrombosis is portal hypertension with recurrent variceal bleeding. Once endoscopic variceal ligation fails and the spleno-portal axis is not permeable, modified Sugiura devascularization may be the only alternative. Its use in patients with cirrhosis has been reported, but there is little information on non-cirrhotic patients. This article presents a series of four cases of non-cirrhotic patients that underwent this procedure. Patients were followed for twelve months; none presented episodes of esophageal varices re-bleeding nor required ligation of residual varices. This surgery is outlined as a therapeutic alternative for this type of patients.

\section{Keywords}

Thrombosis, portal vein, extrahepatic; Portal hypertension; Modified Sugiura; Esophageal varices; Cirrhosis.

\section{INTRODUCCIÓN}

La devascularización ácigos-portal fue introducida en la literatura en 1973 por el Dr. Sugiura (1). Esta consiste en realizar la ligadura de las venas penetrantes del esófago distal y del fondo gástrico, para lo cual se seccionan la vena gástrica izquierda y la vena gastroepiploica a nivel del inicio de la curvatura mayor, al igual que los vasos cortos. Este procedimiento incluía, inicialmente, la sección del esófago y la posterior anastomosis término-terminal, así como una piloroplastia, una esplenectomía y una vagotomía selectiva.

En las primeras descripciones, ya se reportaba la efectividad de este procedimiento, con una incidencia de resangrado $<1,5 \%$, así como con una sobrevida a los 10 años $>70$ $\%$ en los pacientes programados y $>50 \%$ en los urgentes (2). Sin embargo, la falla de la anastomosis del esófago se asoció a muchas complicaciones y a una tasa de mortalidad significativa. Por tal razón, la técnica se modificó y se 
excluyó, en principio, la sección del esófago, y luego la vagotomía y la piloroplastia (3). En la Figura 1 se muestran las diferencias entre la técnica original y la técnica modificada.

Una vez se realiza el procedimiento, el paciente es seguido por medio de endoscopias y la ligadura de várices residuales o de nueva aparición. Algunos estudios han mostrado un control de más del $80 \%$ del resangrado a 5 años, por lo que esta podría ser una buena alternativa terapéutica para los pacientes en los cuales el manejo con bloqueante $\beta$ y ligadura no son suficientes, a fin de controlar los episodios de hemorragias recurrentes (4).

La trombosis portal extrahepática tiene una prevalencia cercana a 5 casos por cada 10000 habitantes, según la Organización Mundial de la Salud (OMS) (5). En el 60 \% de estos casos, se logra identificar una patología hematológica subyacente, mientras que el $40 \%$ restante tiene diversas etiologías (6). Algunos factores de riesgo detectados son las infecciones gastrointestinales, la cateterización umbilical neonatal, los síndromes mieloproliferativos, los anticonceptivos, el embarazo y ciertas cirugías abdominales.

De cualquiera manera, no existen muchas investigaciones acerca del manejo del sangrado variceal en el contexto de esta patología. Las recomendaciones actuales se basan en datos de pacientes con hipertensión portal y cirrosis (7). Este estudio es una serie de casos de pacientes con trombosis portal extrahepática, realizado en aquellos no cirróticos con sangrado variceal recurrente, quienes fueron sometidos a la devascularización de Sugiura modificado.

\section{DESCRIPCIÓN DE LOS CASOS}

Entre 2014 y 2017, en nuestro servicio se manejaron 6 pacientes no cirróticos, con trombosis de la vena porta extrahepática y sangrado recurrente de las várices esofágicas, a pesar de tener ligaduras a repetición y manejo con bloqueantes $\beta$ y terlipresina. De estos pacientes, 2 tuvieron una anatomía susceptible de derivaciones clásicas, y en ellos se realizó una derivación esplenorrenal.

Los 4 pacientes restantes presentaron episodios de sangrado variceal recurrente, con requerimientos de múltiples transfusiones y hospitalizaciones. Al momento de ser evaluados, 3 se encontraban en la unidad de cuidados intensivos (UCI), extubados y recibiendo terlipresina, mientras que 1 permaneció en hospitalización general. Asimismo, 3 de ellos habían recibido múltiples transfusiones y al menos 2 intentos de control del sangrado por endoscopia durante
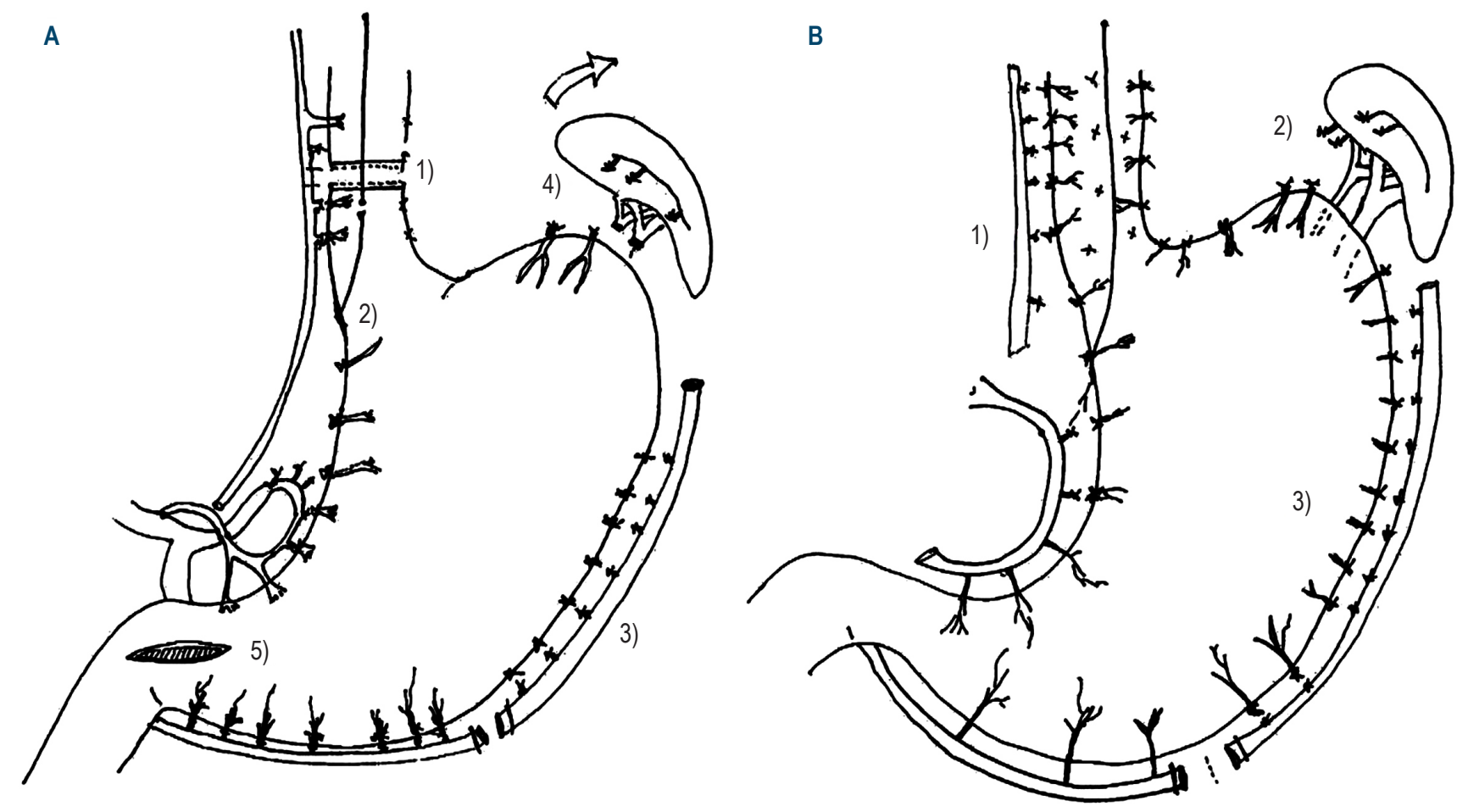

Figura 1. Técnica de Sugiura original frente a la técnica modificada. A. Técnica de Sugiura original: 1) transección del esófago y sección del nervio vago; 2) devascularización de la curvatura menor y del esófago distal; 3) devascularización de la curvatura mayor; 4) esplenectomía; 5) piloroplastia. B. Técnica de Sugiura modificada: 1) devascularización de la curvatura menor y del esófago distal;2) ligadura de los vasos cortos; 3) devascularización de la curvatura mayor. 
el último mes. El otro individuo había sufrido el episodio más reciente 5 semanas antes.

Durante el protocolo de evaluación, se realizó una tomografía dinámica abdominopélvica, con inyección de contraste, a todos los pacientes, además de una esplenoportografía transesplénica combinada y una venografía por vía femoral a 2 pacientes. Inicialmente, se evaluó la posibilidad de realizar una derivación convencional tipo esplenorrenal, mesocava o meso-Rex. La falta de dilatación de la vena esplénica o la trombosis de esta, o la distancia entre la vena renal y la esplénica, impidieron en todos los casos la realización de una derivación esplenorrenal.

Asimismo, la ausencia de permeabilidad de la vena mesentérica superior, con trombosis clasificada de grado IV, según Yerdel (8), impidió el uso de esta estructura como partida para una derivación hacia la cava o hacia la porta izquierda intrahepática.

De igual manera, 3 de los pacientes fueron operados en el contexto de urgencia por hemorragia, y el cuarto fue sometido a una cirugía programada, pues llevaba varias semanas sin sangrado activo. En todos los pacientes, el servicio de hematología descartó la existencia de enfermedades trombofílicas.

Se realizó el procedimiento de Sugiura modificado, reportado en la literatura, con un abordaje abdominal único, mediante incisión en la línea media supraumbilical, ligadura de los vasos penetrantes de los tercios proximales del estómago, disección y ligadura de todas las venas que penetran el esófago distal. Además, se usó la técnica transhiatal -al menos $7 \mathrm{~cm}$ intratorácicos- y se efectuó la disección hasta las capas musculares distales para lograr la ligadura de las várices y la sección de la vena coronaria izquierda y de los vasos gastroepiploicos izquierdos, a nivel de la transición del antro a la curvatura mayor.

También se seccionaron los vasos cortos y se utilizaron dispositivos de energía en vasos $<5 \mathrm{~mm}$ y ligaduras con seda 000 y 00 . No se realizó la esplenectomía ni la ligadura de la arteria esplénica, y tampoco se efectuó la sección del esófago, la piloroplastia, ni la vagotomía selectiva.

El promedio de tiempo quirúrgico fue de $2,5 \mathrm{~h}$ y ninguno de los pacientes recibió vasoactivos durante el procedimiento. Entre tanto, el sangrado promedio durante las intervenciones fue de $200 \mathrm{~cm}^{3}$, sin llegar a requerirse de transfusiones por sangrado intraoperatorio. Los 4 pacientes fueron extubados en cirugía y trasladados a la UCI, con una hemodinamia estable. Durante el posoperatorio se inició progresivamente la vía oral, sin uso de sondas de nutrición. A continuación, se describen las principales características de estos pacientes (Tabla $\mathbf{1}$ ).

Durante el período posoperatorio, una paciente presentó ascitis transitoria, la cual se resolvió con diuréticos, mientras que otro de los enfermos tuvo vaciamiento gástrico retardado, que se corrigió sin tratamiento. No hubo reinterven- ciones ni colecciones residuales. Todos los pacientes alcanzaron los requerimientos calóricos por vía oral y fueron dados de alta con orden de seguimiento y endoscopias seriadas a los 8 días, así como en los meses 1, 3, 6 y 12. Los hallazgos endoscópicos están resumidos a continuación (Tabla 2).

Tabla 1. Características de los pacientes

\begin{tabular}{lcccc}
\multicolumn{1}{c}{ Características } & \multicolumn{4}{c}{ Casos } \\
\cline { 2 - 5 } & $\mathbf{1}$ & $\mathbf{2}$ & $\mathbf{3}$ & $\mathbf{4}$ \\
\hline Edad (años) & 24 & 68 & 36 & 42 \\
\hline Sexo (F o M) & F & F & M & M \\
\hline Factores de riesgo & $\begin{array}{c}\text { Cateterismo } \\
\text { umbilical }\end{array}$ & Diverticulitis & Ninguno & Ninguno \\
$\begin{array}{l}\text { UCl por HVDA } \\
\text { Transfusión }>4 \text { UGRE } \\
\text { en los últimos 2 meses }\end{array}$ & Sí & Sí & Sí & Sí \\
$>3$ ligaduras & Sí & Sí & Sí \\
$\begin{array}{l}\text { Hemorragia reciente } \\
\text { (<15 d) }\end{array}$ & Sí & Sí & Sí & Sí \\
\hline
\end{tabular}

F: femenino; M: masculino; UCI: unidad de cuidados intensivos; HVDA: hemorragia de vías digestivas altas; UGRE: unidades de glóbulos rojos empaquetados

Tabla 2. Grado de várices esofágicas a los 3, 6 y 12 meses, según la clasificación de Dagradi

\begin{tabular}{ccccc|} 
& \multicolumn{3}{c|}{ Grado de várices esofágicas } \\
\cline { 2 - 5 } Paciente & 3 meses & $\mathbf{6}$ meses & 12 meses \\
\hline 1 & $\|$ & $\|$ & 0 \\
2 & 1 & 1 & 1 \\
3 & 0 & 0 & 1 \\
4 & 0 & 1 & 1
\end{tabular}

Dentro de ese contexto, en el control de los 3 meses, solo 2 pacientes tuvieron várices residuales grados I y II $(<5$ $\mathrm{mm}$ ), mientras que en el primer paciente desaparecieron mediante la insuflación. Los otros 2 individuos no presentaron várices en el esófago ni en el fondo gástrico, aunque sí se observó la existencia de gastropatía hipertensiva portal.

No hubo necesidad de ligaduras profilácticas, y a todos los pacientes se les retiró el bloqueante $\beta$, excepto a aquella con várices grado II, la cual presentó un sangrado por várices colónicas luego de un mes de la operación. Estas se manejaron de forma conservadora, con el aumento del bloqueante $\beta$. No se documentó un resangrado en el seguimiento después de 8 meses. 
$\mathrm{Al}$ año de control, 3 pacientes tuvieron várices $<5 \mathrm{~mm}$, que desaparecieron con insuflación, mientras que el cuarto individuo no presentó ninguna manifestación varicosa. De igual forma, ninguno de los pacientes mostró resangrado de las várices esofágicas.

\section{DISCUSIÓN}

Existen reportes sobre los resultados de esta operación en términos del control del resangrado variceal. Sin embargo, la mayoría de ellos han sido realizados en el contexto de pacientes con cirrosis y trombosis de todo el eje esplenoportal. Dichos pacientes fueron conducidos a devascularización, dado que un trasplante de hígado no era técnicamente posible y el trasplante multivisceral no estaba disponible (3).

En contraste, las complicaciones de la hipertensión portal por trombosis de la vena porta extrahepática, no asociada a cirrosis, han sido poco descritas. En caso de falla del manejo médico y endoscópico, la realización de derivaciones (shunts) quirúrgicas o percutáneas es el tratamiento de elección. Sin embargo, estas opciones son limitadas, puesto que hasta en el $50 \%$ de los casos se ha observado trombosis de las venas esplénica y mesentérica superior. El procedimiento de Sugiura modificado surge entonces como una opción para estos pacientes (7, 9-11).

En Japón se han descrito reportes que muestran un control del resangrado que supera el $70 \%$ a los 10 años y el
$80 \%$ a los 5 años (12). Por el contrario, algunos estudios, como el publicado por Mercado y colaboradores, no revelan buenos resultados a largo plazo (13).

Así bien, los 4 pacientes evaluados en este estudio presentaron un cuadro clínico severo por la recurrencia del sangrado variceal, además de múltiples hospitalizaciones y transfusiones. Hasta el momento de la escritura de este artículo, ninguno de ellos ha requerido de la ligadura de várices y el único resangrado ocurrió por várices colónicas. Ningún paciente tiene signos de disfunción hepática y todos han recuperado su actividad laboral. Por tanto, un seguimiento periódico con endoscopia permite la detección temprana de las várices residuales y la necesidad de ligadura.

En nuestra experiencia, el procedimiento de Sugiura modificado puede ser una opción segura para controlar el riesgo de resangrado en los pacientes con hemorragias variceales recurrentes y con trombosis del eje esplenomesentérico extrahepático -de origen no cirrótico- y que, además, no son candidatos a otras terapias convencionales como las derivaciones portosistémicas. Son necesarios estudios con mayor tamaño de muestra y seguimiento a largo plazo para fortalecer el conocimiento en esta área.

\section{Fuente de financiación}

Declaramos no haber recibido ninguna financiación para la realización de este estudio.

\section{REFERENCIAS}

1. Sugiura M, Futagawa S. A new technique for treating esophageal varices. J Thorac Cardiovasc Surg. 1973;66(5):677-685. https://doi.org/10.1016/S0022-5223(19)40560-6

2. Sugiura M, Futagawa $S$. Esophageal transection with paraesophagogastric devascularizations (the Sugiura procedure) in the treatment of esophageal varices. World J Surg. 1984;8(5):673-679. http://doi.org/10.1007/BF01655762

3. Voros D, Polydorou A, Polymeneas G, Vassiliou I, Melemeni A, Chondrogiannis K, Arapoglou V, Fragulidis GP. Longterm results with the modified Sugiura procedure for the management of variceal bleeding: standing the test of time in the treatment of bleeding esophageal varices. World J Surg. 2012;36(3):659-66. http://doi.org/10.1007/s00268-011-1418-7

4. Schettini AV, Pinheiro RS, Pescatore P, Lerut J. Modified Sugiura Operation for Idiopathic Portal Hypertension with Bleeding Oesophageal Varices. A Case Report. Acta Chir Belg. 2015;115(3):237-240.

http://doi.org/10.1080/00015458.2015.11681105
5. Garcia-Pagán JC, Hernández-Guerra M, Bosch J. Extrahepatic portal vein thrombosis. Semin Liver Dis. 2008;28(3):282-292. http://doi.org/10.1055/s-0028-1085096

6. Valla DC, Condat B. Portal vein thrombosis in adults: pathophysiology, pathogenesis and management. J Hepatol. 2000;32(5):865-871. http://doi.org/10.1016/s0168-8278(00)80259-7

7. Llop E, Seijo S. Treatment of non-cirrhotic, nontumoural portal vein thrombosis. Gastroenterol Hepatol. 2016;39(6):403-410. http://doi.org/10.1016/j.gastrohep.2015.09.007

8. Yerdel MA, Gunson B, Mirza D, Karayalçin K, Olliff S, Buckels J, Mayer D, McMaster P, Pirenne J. Portal vein thrombosis in adults undergoing liver transplantation: risk factors, screening, management, and outcome. Transplantation. 2000;69(9):1873-81. http://doi.org/10.1097/00007890-200005150-00023

9. Grace ND. Prevention of initial variceal hemorrhage. Gastroenterol Clin North Am. 1992;21(1):149-161. 
10. de Franchis R, Primignani M. Endoscopic treatments for portal hypertension. Semin Liver Dis. 1999;19(4):439-455. http://doi.org/10.1055/s-2007-1007131

11. Soonawalla ZF, Shah SR, Mathur SK. Modified sugiura procedure. 2002;194(2):247. https://doi.org/10.1016/S1072-7515(01)01146-2

12. Dagenais M, Langer B, Taylor BR, Greig PD. Experience with radical esophagogastric devascularization procedures (Sugiura) for variceal bleeding outside Japan. World J Surg.
1994; 18(2):222-8.

http://doi.org/10.1007/BF00294405

13. Mercado MA, Orozco H, Vasquez M, Pantoja JP, Contreras A, Catzin-Kuhlmann A, Flores A, Rodriguez-Davalos M. Comparative study of 2 variants of a modified esophageal transection in the Sugiura-Futagawa operation. Arch Surg. 1998;133(10):1046-9.

http://doi.org/10.1001/archsurg.133.10.1046 